\title{
INDO-PACIFIC BIODIVERSITY OF CORAL REEFS: DEVIATIONS FROM A MID-DOMAIN MODEL
}

\author{
Sean R. Connolly, ${ }^{1}$ David R. Bellwood, and Terence P. Hughes \\ Centre for Coral Reef Biodiversity, Department of Marine Biology, James Cook University, Townsville, \\ Queensland 4811 Australia
}

\begin{abstract}
Understanding the nature and causes of global gradients in species richness is a perennial ecological problem, and recent work has highlighted the need to assess these gradients relative to an appropriate statistical expectation. This paper examines latitudinal and longitudinal gradients in species richnesses of corals and reef fishes in the Indo-Pacific domain and compares them with gradients predicted by a mid-domain model in which geographic domains are located at random between the latitudinal and longitudinal boundaries of this region. We test for significant differences between observed and predicted species-richness patterns, and we identify regions that are enriched or depauperate in species, relative to expectation. In addition, we move beyond previous mid-domain analyses by directly comparing observed spatial distributions of geographic ranges with those predicted by a mid-domain model. This comparison indicates precisely how species-richness anomalies are produced by nonrandomness in the distribution of species ranges. For both corals and fishes, large and statistically significant differences exist between observed latitudinal and longitudinal species-richness gradients and those predicted by mid-domain models. Longitudinally, species richness is markedly higher than predicted along the African coast and, to a lesser extent, within the Indo-Australian Archipelago (IAA), and it is markedly lower than expected in the eastern Pacific. Latitudinally, species richness becomes increasingly higher than predicted as one moves from the equator to the tropical margins; then it becomes sharply lower than predicted beyond the tropics. Unexpectedly, differences between observed and predicted spatial distributions of range endpoints and midpoints reveal a pattern of nonrandomness that is highly congruent with the hypothesis that gyres in the Indian and Pacific Oceans, with the IAA forming a porous boundary between them, have a major influence on Indo-Pacific species-richness patterns. Our analyses indicate that the perspective offered by a focus on explaining nonrandomness in the location of geographic ranges (rather than explaining why species numbers vary in space) is likely to dramatically alter our assessments of alternative explanations for global species-richness gradients.
\end{abstract}

Key words: coral reefs; geographic range; Indo-Australian Archipelago (IAA); latitudinal gradient; longitudinal gradient; macroecology; mid-domain effect.

\section{INTRODUCTION}

Global-scale patterns in species richness have engaged ecologists since the dawn of modern ecological and evolutionary thinking (e.g., Wallace 1878). The best-known and most widely studied pattern is the latitudinal gradient in species richness (Pianka 1978, Huston 1994, Rosenzweig 1995). Like most nearshore marine taxa, coral reef organisms generally exhibit a longitudinal biodiversity gradient: species richness declines with increasing distance from the Indo-Australian Archipelago (IAA), a triangular region bounded by Sumatra in the west, Papua New Guinea/Solomon Islands in the southeast, and the Philippines in the north (approximately $90^{\circ}-160^{\circ} \mathrm{E}, 10^{\circ} \mathrm{S}-15^{\circ} \mathrm{N}$; Stehli and Wells 1971, Roberts et al. 2002). Several hypotheses

Manuscript received 26 April 2002; revised 14 November 2002; accepted 2 December 2002. Corresponding Editor: N. J. Gotelli.

${ }^{1}$ E-mail: sean.connolly@jcu.edu.au have been proposed to explain these patterns (Rosen 1988, Paulay 1997, Bellwood and Hughes 2001).

Geographical gradients in species richness arise from geographical patterns in the rates at which species originate, new populations are founded by dispersal (i.e., ranges expand), and existing populations become extinct. Each of these processes has been invoked in explanations for Indo-Pacific species-richness gradients. "Center of origin" theories hypothesize that speciation rates are unusually high within the IAA, and that this is responsible for high biodiversity in this region. Gradual latitudinal and longitudinal declines in species richness with increasing distance from the IAA are attributed to variation in the extents to which species subsequently expand their ranges outward from this region. Particular mechanisms invoked have included elevated speciation driven by high rates of solar energy input (sensu Rohde 1992), abundant habitat area (Bellwood and Hughes 2001), or vicariance induced by glacioeustatic sea-level fluctuations (McManus 1985). Dis- 
persal-related hypotheses attribute high diversity in the IAA to a tendency for species of Pacific origin to extend their geographic ranges towards this region, given the tendency for equatorial currents to flow from east to west across the Pacific Ocean (Ladd 1960, Jokiel and Martinelli 1992). Finally, extinction-related hypotheses attribute high diversity in the IAA to lower extinction rates there, either presently or at some time in the past. Larger population sizes or greater potential for resource partitioning owing to abundant habitat area or higher energy input are plausible reasons to expect reduced extinction in the IAA (Rosen 1988, Fraser and Currie 1996, Palumbi 1997, Bellwood and Hughes 2001). An hypothesized tendency for small-range endemics with narrow environmental tolerances to be concentrated in the IAA — and thus for mean range size to increase with increasing distance from the center of biodiversity-is another possible explanation for latitudinal and longitudinal species-richness gradient (sensu Rapoport's Rule; Stevens 1989). Opinions differ about the extent to which endemics are concentrated in the IAA (Veron 1995, Jones et al. 2002, Roberts et al. 2002). Resolution of this issue has been plagued by statistical problems (see Lyons and Willig 1997). However, a recent analysis that is robust to those problems has shown no tendency for mean range size to increase with increasing distance from the IAA for either corals or reef fishes (Hughes et al. 2002).

Hypotheses that incorporate various combinations of area, energy, and oceanic dispersal processes have proliferated extensively over the past three decades (Rosen 1988, Fraser and Currie 1996, Palumbi 1997, Paulay 1997), with little movement towards a consensus. Here, we begin with an approach that has been used productively in other systems: recasting the problem from one of species-richness patterns, per se, to one of patterns in the location of geographic ranges (see Colwell and Lees 2000 for a review). We formally test the hypothesis that geographic ranges are distributed at random, and that species-richness gradients are artifacts of the bounded nature of the Indo-Pacific latitudinal and longitudinal domains. This test demonstrates highly significant nonrandomness in the observed distributions of geographic ranges, and allows us to identify those regions that are enriched or depauperate, relative to expectation. We then characterize how nonrandomness in the spatial distributions of species ranges deviates from expectation. These deviations provide a fresh perspective on the nature and causes of Indo-Pacific species-richness gradients, and indicate the need for a substantial reassessment of the major alternative hypothesized causes of Indo-Pacific species-richness gradients.

\section{Biodiversity, geographic range, and geometric constraints}

Traditionally, ecologists have assessed explanations for latitudinal and longitudinal biodiversity gradients

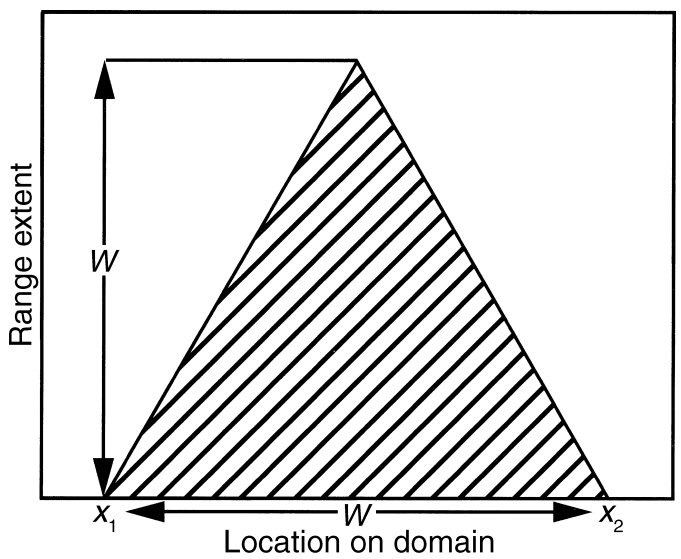

FIG. 1. Geometric constraints on the locations of geographic range, illustrated in a hypothetical plot of range midpoint vs. range size. All geometrically feasible combinations of range midpoint location and range size are contained within the triangle shown. The domain ranges from $x_{1}$ to $x_{2}$, and has total width $W$. Thus, maximum range size is $W$, and this can only be realized if range midpoint is at the center of the domain (i.e., the apex of the triangle). For smaller range sizes (further down the vertical axis) more midpoint locations become possible, with feasible locations for range midpoint approaching the entire width of the domain as range size approaches zero.

by identifying concordant gradients in biological and physical factors that are likely to influence the number of species that a region can support (Huston 1994, Rosenzweig 1995). Implicit in this approach is an assumption that the null expectation-the expected species-richness pattern in the absence of variation in these environmental factors-is similar levels of species richness everywhere. However, a random distribution of geographic ranges produces a peak in species richness midway between the boundaries of a biogeographic domain, and it has been proposed that this "middomain effect" represents the null expectation of species richness (Colwell and Hurtt 1994). Macroecologists have largely accepted this proposal, and comparison of observed biodiversity patterns with predictions based on randomizations of geographic range have become increasingly commonplace (e.g., latitude, Willig and Lyons 1998; depth, Piñeda and Caswell 1998; altitude, Rahbek 1997; two-dimensional space, Jetz and Rahbek 2001).

Mid-domain models begin with the recognition that domain boundaries place geometric constraints on feasible combinations of range extents and locations. These constraints are commonly depicted in a plot of range midpoint location against range extent (Fig. 1). A species is represented in this figure as a point: its horizontal coordinate indicates the location of its midpoint, and its vertical coordinate indicates its range extent. Feasible combinations of midpoint location and range extent are points within a triangle whose base extends horizontally from one domain boundary to the other, and whose maximum height is equal to the total 
domain width. Thus, a pandemic species whose range is as extensive as the total domain width (height, $W$, in Fig. 1) must be centered in the middle of the domain if it is to fit within that domain (i.e., at the apex of the triangle in Fig. 1). As range extent becomes smaller (i.e., as one moves down the triangle), geometrically feasible locations of range midpoint broaden, until finally, as range extent approaches zero, the range midpoint can fall anywhere within the domain (at the base of the triangle).

Mid-domain models are constructed by randomly drawing midpoint locations, range extents, or both from among these geometrically feasible values. There are multiple ways to conduct this randomization. For instance, if all geometrically feasible midpoint-range extent combinations are equally likely, the expected density of midpoints inside the triangle is uniform (Model I of Colwell and Hurtt 1994; the binomial model of Willig and Lyons 1998). Variants of this model include choosing range extent $E$ at random, then randomizing range location $L$, conditional upon the value of $E$, or vice versa (Colwell and Hurtt 1994). Alternatively, one may take the observed distribution of either range extent or range midpoint, and randomize only the other quantity (Piñeda and Caswell 1998).

In this paper, we analyze the extent to which a middomain effect can explain species-richness gradients in Indo-Pacific corals and reef fishes, using a recentlyassembled database of geographic ranges of scleractinian corals and reef fishes in the Indo-Pacific (see Hughes et al. 2002). We model a mid-domain effect by randomizing range locations, given observed range extents. This allows an unambiguous and rigorous test for nonrandom distributions of range locations, in other words, an assessment of whether or not ranges are more clustered in space (e.g., within the IAA center of biodiversity) than expected by chance alone. In addition, we devise and apply two new approaches to directly comparing observed distributions of species ranges to mid-domain predictions. Our results fundamentally redefine the patterns that putative causes of species-richness gradients must explain, so we conclude with a discussion of our findings' implications for three major hypothesized causes of the Indo-Pacific biodiversity gradents: habitat availability, energy input, and oceanic gyres.

\section{Methods}

The database used in our analysis includes the latitudinal and longitudinal endpoints of all 727 zooxanthellate Indo-Pacific tropical reef corals, and 1766 reefassociated fish species from 13 families (Chaetodontidae, Pomacanthidae, Acanthuridae, Siganidae, Scaridae, Pomacentridae, Labridae, Blennidae, Apogonidae, Scorpaenidae, Holocentridae, Serranidae, and Lutjanidae). These families represent all of the major reef fish families for which reliable data on species distributions can be obtained. Sources of data are available elsewhere
(Hughes et al. 2002). To satisfy assumptions of the middomain model, ranges were not truncated. Because some reef-associated fish species extend beyond the limits of coral species, fish domains were slightly more extensive than coral domains. If cryptic speciation is more prevalent in one taxon (e.g., corals) than another (e.g., fishes) species ranges will implicitly incorporate these differences in degree of lumping or splitting between coral and fish taxonomists. This could obviously have a substantial impact on the distribution of range sizes, and thus make it difficult to interpret results of randomizations of range size. However, because our models take observed range sizes as given, and focus on comparing observed and predicted locations of those ranges, our analyses should be relatively robust to any such taxonomic artifacts.

In this paper, we model range location as uniformly distributed among geometrically feasible values, given observed range extents. In other words, we randomize range location, but not range extent (also see Piñeda and Caswell 1998, Veech 2000). Thus, deviations from mid-domain expectation indicate the effects of the geographical clustering of species ranges. From these randomizations, we proceed systematically from a formal statistical comparison of observed species-richness patterns versus a mid-domain prediction, towards a quantitative characterization of what it is about the distribution of geographic ranges that is responsible for the differences between observed and predicted species richness.

When randomizing range location, one may preserve one of two measures of range size (Gaston and Blackburn 2000): extent of occurrence (area encompassed by a species' range), or area of occurrence (actual area occupied by a species). In this paper, we focus on extent of occurrence, and we analyze observed and predicted distributions of geographic range separately along latitudinal and longitudinal axes. This analysis preserves the distance between most distal habitats (the data upon which measures of range extent are based), rather than the shape or area enclosed by the range (which may be sensitive to the way in which the envelope connecting those habitats is drawn). As we will demonstrate, substantial information can be gleaned by examining deviations from one-dimensional expectations, particularly when latitudinal and longitudinal deviations are interpreted together (see Appendix A for further explanation and justification).

There are two key potential sources of bias in such analyses. Firstly, when the geographical domain is irregularly shaped, the longitudinal width of the domain changes with latitude, and the latitudinal width of the domain changes with longitude. Secondly, the analyses allow species' range endpoints to fall anywhere, when, in reality, they are biologically constrained to occur only at latitudes and longitudes where there is habitat in which the species can be present. We relaxed these assumptions by randomizing latitudinal extent given 
the boundary constraints imposed by observed longitudinal extent, randomizing longitudinal extent given the boundary constraints imposed by observed latitudinal extent, and constraining range endpoints to fall only where there was suitable shallow-water habitat (Appendix B). We then repeated all of our analyses to assess the extent to which our results may be sensitive to those assumptions.

To test for significant departure from mid-domain predictions, we use randomizations to generate both a predicted species-richness curve, and the expected distribution of a displacement statistic, $D$, which measures the extent to which any observed species-richness curve would be expected to deviate from the predicted curve by chance alone (Veech 2000). Results reported here are based on an analysis using 1000 randomizations. We then examine differences between observed and predicted species richnesses as functions of latitude and longitude, identifying regions that are significantly enriched or depauperate relative to expectation.

Veech's $D$ allows for an unambiguous test for significant differences between observed and expected species-richness patterns in the aggregate. We also analyze the distribution of range endpoints and midpoints directly to understand how deviations between observed and predicted species richnesses arise. Because of geometric constraints, ranges that encompass most of the domain must be centered near the domain center, and have endpoints close to the domain boundaries. Thus, one would expect more endpoints near domain boundaries than near the mid-domain; conversely, one would expect more midpoints near the mid-domain than towards the boundaries. Here, we quantitatively characterize these expected distributions of endpoints and midpoints, and we compare them with the observed distributions. This comparison indicates regions where there is a paucity or accumulation of range endpoints or midpoints relative to the patterns expected under a mid-domain effect alone.

A larger-than-predicted accumulation of midpoints or endpoints at a particular location may be due to excess small-range endemics, or to excess medium or large range species that end, or are centered, at that location. Species range locations and sizes are often jointly examined with "midpoint plots," plots of range extent vs. midpoint location within the triangle shown in Fig. 1 (e.g., Colwell and Hurtt 1994). We exploit this analytical tool here, and systematically compare midpoint plots of the observed data with those expected under a mid-domain effect alone. We do this from our mid-domain randomizations by identifying those regions of the triangle for which observed densities of midpoints lie above, below, and within confidence limits that mark the boundaries between which $99 \%$ of the randomized midpoint densities fall.

\section{RESULTS}

Species-richness patterns

Longitudinally, coral and fish species-richness patterns are highly asymmetric, and thus differ markedly from mid-domain predictions (Fig. 2A, C). Latitudinally, agreement between observed and predicted biodiversity appears much closer (Fig. 2B, D). The eye is drawn to two shared features of observed and predicted species richness in the latitudinal plots: their peaks, which occur at or near the mid-domain and are of comparable magnitude, and their symmetric, quasi-parabolic, decreases towards zero at the domain boundaries. However, this apparent agreement between observed and predicted is deceptive: mean displacement, $D$, is highly significant for latitude and longitude, and for both corals and fishes $(P<0.001$ in every case).

Normalizing observed species richness to that predicted by the mid-domain model reveals that the differences between observed and predicted species richness are large, on the order of hundreds of species in some regions (Fig. 3). Longitudinally, deviations of coral and fish species richness are qualitatively similar (Fig. 3A). There are two peaks of higher-than-predicted diversity. The largest occurs at $\sim 33^{\circ} \mathrm{E}$, corresponding to the easternmost limit of the Red Sea and the coast of southern Mozambique. A second peak occurs in the longitudes straddling the Indian and Pacific Oceans (Australia, the Indo-Australian Archipelago or "IAA," and north to southern Japan, hereafter "longitudes of the IAA"). For corals, this anomaly is highest at the region's western margin $\left(\sim 90^{\circ} \mathrm{E}\right)$. For fishes, it is further east $\left(\sim 145^{\circ} \mathrm{E}\right)$. There is also a negative anomaly in that species richness is lower than expected throughout the Pacific, bottoming out just east of French Polynesia, near $135^{\circ} \mathrm{W}$. It is noteworthy that the IAA biodiversity hotspot is indeed enriched relative to expectation even though it lies near the center of the domain. However, it is also striking that the higherthan-expected diversity near Africa, and the lowerthan-expected diversity in the mid-Pacific, are actually slightly farther from expectations than the diversity anomaly in the longitudes of the IAA. This is true for both corals and fishes (compare magnitudes of peaks and troughs in Fig. 3A).

In contrast to longitude, latitudinal deviations in species richness of corals and fishes are qualitatively different from one another (Fig. 3B). Coral species richness tends to be higher than expected north of the equator, but lower than expected south of it. By contrast, fish species richness is lower than expected in both the northern and southern subtropics; diversity shifts abruptly to higher than expected with the transition to the tropics; then the anomaly declines towards its expected value near the equator. Again, it is striking that positive deviations in species richness do not peak near the equator, but rather to the north (for corals and fishes), and south (for fishes), beyond the equatorial latitudes of the high-diversity IAA.

\section{Spatial distributions of endpoints and midpoints}

Comparing observed locations of range endpoints with those expected under a mid-domain effect alone 


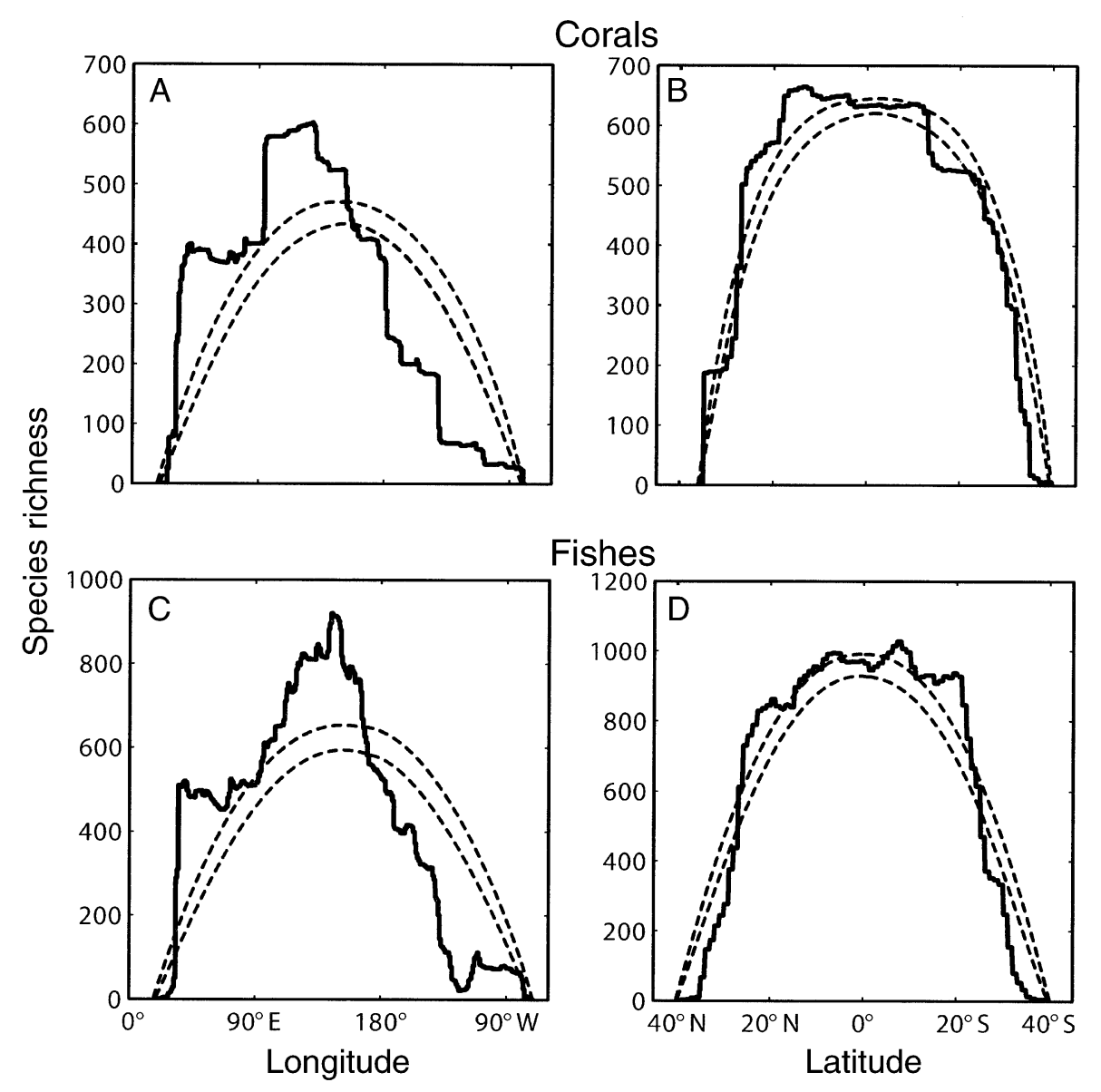

FIG. 2. Observed longitudinal and latitudinal species-richness patterns, plotted with predicted values under the middomain model: (A) corals, longitude; (B) corals, latitude; (C) fish, longitude; and (D) fish, latitude. Dashed lines encompass 99\% of the species-richness values obtained from the randomizations (i.e., all but the lower and upper $0.5 \%$ of randomized species-richness values). Species richness is calculated as the number of species whose ranges encompass a particular longitude $(\mathrm{A}, \mathrm{C})$ or latitude $(\mathrm{B}, \mathrm{D})$.

helps to identify how these species-richness deviations arise (Fig. 4). Longitudinally, the largest discrepancy between observed and expected endpoints occurs at the western boundary of the Indo-Pacific (Fig. 4A, C). For corals, over 200 species more than expected end their ranges along the African coast (Fig. 4A); for fishes, the excess climbs to more than 300 species (Fig. 4C). Proportionally, these numbers represent more than twice the expected number of species, for both corals and fishes. There is an additional excess of western range limits within the longitudes of the IAA. For corals, this excess occurs near Sumatra, at the western margin of the IAA (Fig. 4A). For fishes, excess western endpoints occur throughout the region (Fig. 4C). There is a dearth of western limits elsewhere, except for a small positive anomaly for fishes, representing eastern Pacific endemics (far right of Fig. 4C). By contrast, there is a paucity of eastern endpoints along the eastern margin of the Indo-Pacific, with corals and fishes instead tending to reach their eastern limits across a broad region from the eastern IAA to French Polynesia (Fig. 4A, C). Lat- itudinally, agreement between observed and predicted endpoint distributions is closer. In the north, there is an excess of coral and fish species with northern endpoints falling between $25^{\circ}$ and $30^{\circ} \mathrm{N}$, and a corresponding paucity just north and south of this region (Fig. 4B, D). For fishes, this pattern is closely mirrored in the Southern Hemisphere (Fig. 4D); corals show a smaller excess of southern endpoints located closer to the equator $\left(10^{\circ}-15^{\circ} \mathrm{S}\right.$, Fig. $\left.4 \mathrm{~B}\right)$.

Range midpoint patterns of corals and fishes show very similar patterns (Fig. 5). Longitudinally, there is a marked excess of midpoints throughout the longitudes of the IAA, and a smaller excess of midpoints near the western margin of the Indo-Pacific (Fig. 5A, C). Latitudinally, more species are centered near the equator than expected under a mid-domain effect alone (Fig. 5B, D). Corals and fishes both show a small secondary excess near the northern margin of the tropics $\left(15^{\circ}-20^{\circ} \mathrm{N}\right.$, Fig. 5B, D). Fishes show an additional, and larger, accumulation to the south $\left(20^{\circ}-25^{\circ} \mathrm{S}\right.$, Fig. 5D). 

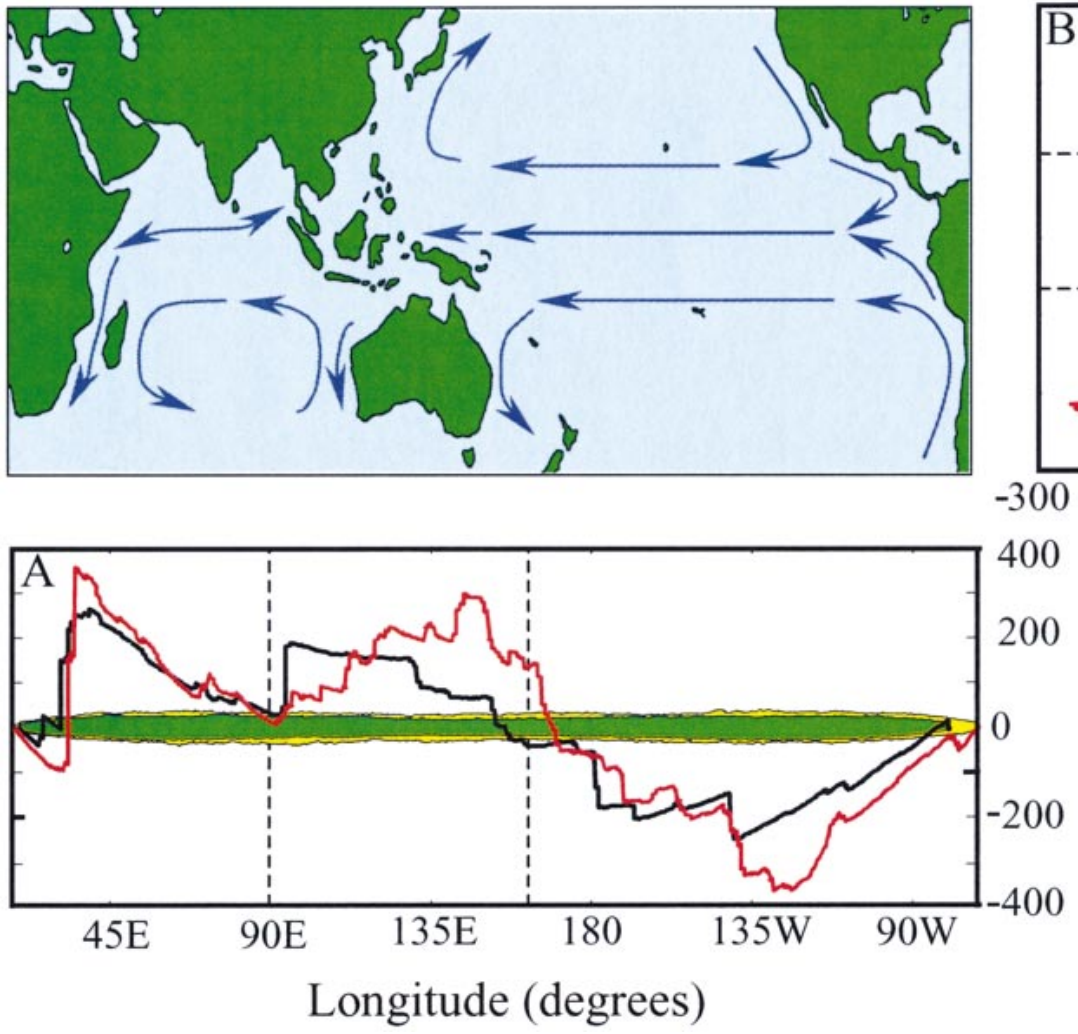

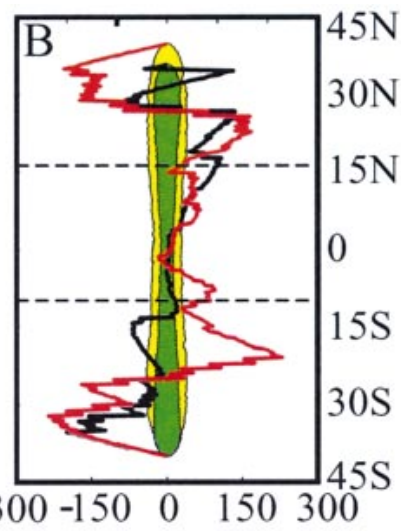

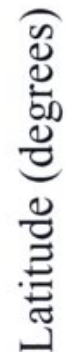

FIG. 3. (A) Longitudinal and (B) latitudinal species-richness patterns for corals (black lines) and fish (red lines), expressed as deviations from species richness predicted by the mid-domain model. Shaded areas encompass the range of deviations expected by chance alone, calculated from randomizations as described in Fig. 2. Yellow shading indicates fish confidence regions, and green shading indicates coral confidence regions. Dashed lines represent the boundaries of the Indo-Australian Archipelago. Arrows on the Indo-Pacific map indicate the direction of major surface currents, and the double-headed arrow in the northern Indian Ocean indicates a seasonal (monsoonal) reversal in flow (after Tomczak and Godfrey 1994). Species richness is calculated as the number of species whose ranges encompass a particular longitude (A) or latitude (B).

\section{Midpoint deviations}

Plots of range midpoint vs. range extent show that the observed data deviate from mid-domain expectation in very similar ways for corals and fishes (Fig. 6). Moreover, as indicated by the prevalence of regions where midpoint densities are above or below the $99 \%$ confidence limits (white and black areas, respectively, Fig. 6), these deviations tend to be very large throughout the domain. Longitudinal midpoints show two striking similarities between corals and fishes. First, there is a higher-than-expected concentration of midpoints of species with large range extents that is adjacent and parallel to the left margin of the triangle, i.e., located as far west as possible, given their range extents (area 1 in Fig. 6A, C). Thus, these species reach their western limits along the African coast, and their large range extents $\left(>100^{\circ}\right.$ longitude) indicate that they span the entire Indian Ocean. Secondly, there is a smaller cluster of species with small longitudinal extents, also centered near the westward geometric constraint (area 2 in Fig. $6 \mathrm{~A}, \mathrm{C}$ ). These are western Indian Ocean and Red Sea endemics, responsible for the small accumulation of range midpoints near the western boundary of the IndoPacific (Fig. 5A, C). Together, these species account for the excess of western limits along the African coast (Fig. 4A, C). Corals show an additional region of higher-than-expected midpoint density (area 3 in Fig. 6A). The diagonal orientation of this area, parallel to the westward boundary constraint, indicates species with a shared westward limit, in this case, Sumatra, at the westward edge of the IAA. This accounts for the corresponding accumulation of western endpoints (Fig. 4A). Fish show two additional clusters. One consists of small-range species, centered across a broad region spanning the longitudes of the IAA (area 4 in Fig. 6C). Another identifies Eastern Pacific endemics (area 5 in Fig. 6C).

This analysis indicates how species with narrow and broad range extents contribute differently to the endpoint and midpoint patterns shown in Figs. 4 and 5 . For corals and fishes, a major contributor to the excess of midpoints in the longitudes of the IAA (Fig. 5A, C) are broad-ranging species that extend from Africa eastward across most of the longitudinal domain (species 


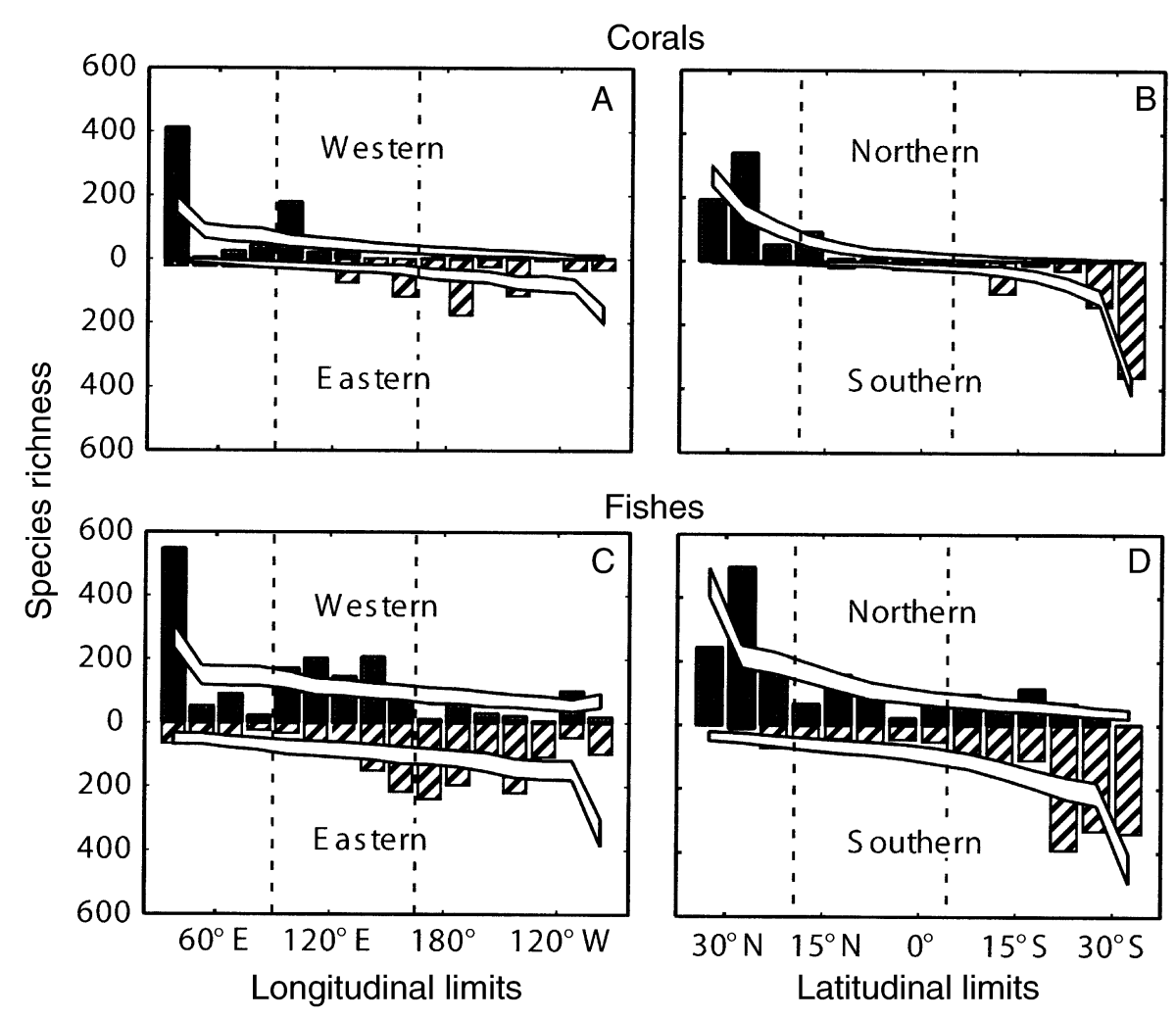

FIG. 4. Observed distribution of range endpoints (bars), and expected distribution based on mid-domain randomizations: (A) corals, longitudinal limits; (B) corals, latitudinal limits; (C) fish, longitudinal limits; and (D) fish, latitudinal limits. Enclosed white regions encompass $99 \%$ of randomizations. In (A) and (C), the upper half of each panel depicts western limits, and the lower half depicts eastern limits. Vertical dashed lines represent the boundaries of the Indo-Australian Archipelago. The leftmost (western) bin includes all endpoints west of $45^{\circ} \mathrm{E}$ (approximately the mouth of the Red Sea and northern Mozambique), while the rightmost bin includes all endpoints east of $105^{\circ} \mathrm{W}$ (central and South America, including the Galápagos Islands). Intervening bins show $15^{\circ}$ intervals. In (B) and (D), the upper half of each panel depicts northern limits, and the lower half depicts southern limits. The leftmost and rightmost bins represent locations north of $30^{\circ} \mathrm{N}$ and south of $30^{\circ} \mathrm{S}$, respectively. Intervening bins show $5^{\circ}$ intervals.

from area 1 in Fig. 6A, C). For corals, an additional cause of the excess of IAA midpoints are species of intermediate extent that extend from Sumatra eastward into the various points in the IAA and western Pacific (species from area 3 in Fig. 6A). For fishes, an additional cause are species with narrow range extents that are centered throughout the central and eastern IAA (area 4 in Fig. 6C).

For latitude, corals and fishes with broad range extents are more centered at the equator than expected, indicated near the apices of the triangle by a region of higher-than-expected midpoint density centered at the equator (area 6 in Fig. 6B, D), flanked by regions of lower-than-expected midpoint density. Because there is no corresponding equatorial excess of species with narrow range extents (Fig. 6B, D), the midpoint excess near the equator (Fig. 5B, D) must be driven by largerange species, not small-range endemics. Rather, smallrange endemics are clustered near tropical margins. For both corals and fishes, species with narrow range extents are more centered than expected near $25^{\circ} \mathrm{N}$ (area 7 in Fig. 6B, D). These species are primarily high lat- itude endemics (principally from the Red Sea, but also from Japan, Hawaii, and Baja California), and they are responsible for the small midpoint excesses near the margin of the northern tropics (Fig. 5B, D). Fishes show an additional excess of small-range species near $20^{\circ} \mathrm{S}$ (area 8 of Fig. 6D). Inspection of the data suggests that these consist principally of South Pacific species found between the Great Barrier Reef and French Polynesia; these species drive the midpoint excess near the southern margin of the tropics (Fig. 5D).

In summary, species with large longitudinal extents extend all the way to Africa more frequently than expected; species with intermediate extents are unusually likely to extend westward to Sumatra (corals) or be centered in the IAA (fish); and species with narrow longitudinal extents are more likely to be found along the coast of Africa (corals and fish), within the IAA (fish), and in the East Pacific (fish) than expected under a mid-domain model. Species with large latitudinal extents are more centered at the equator than expected, while species with narrow latitudinal extents are clus- 


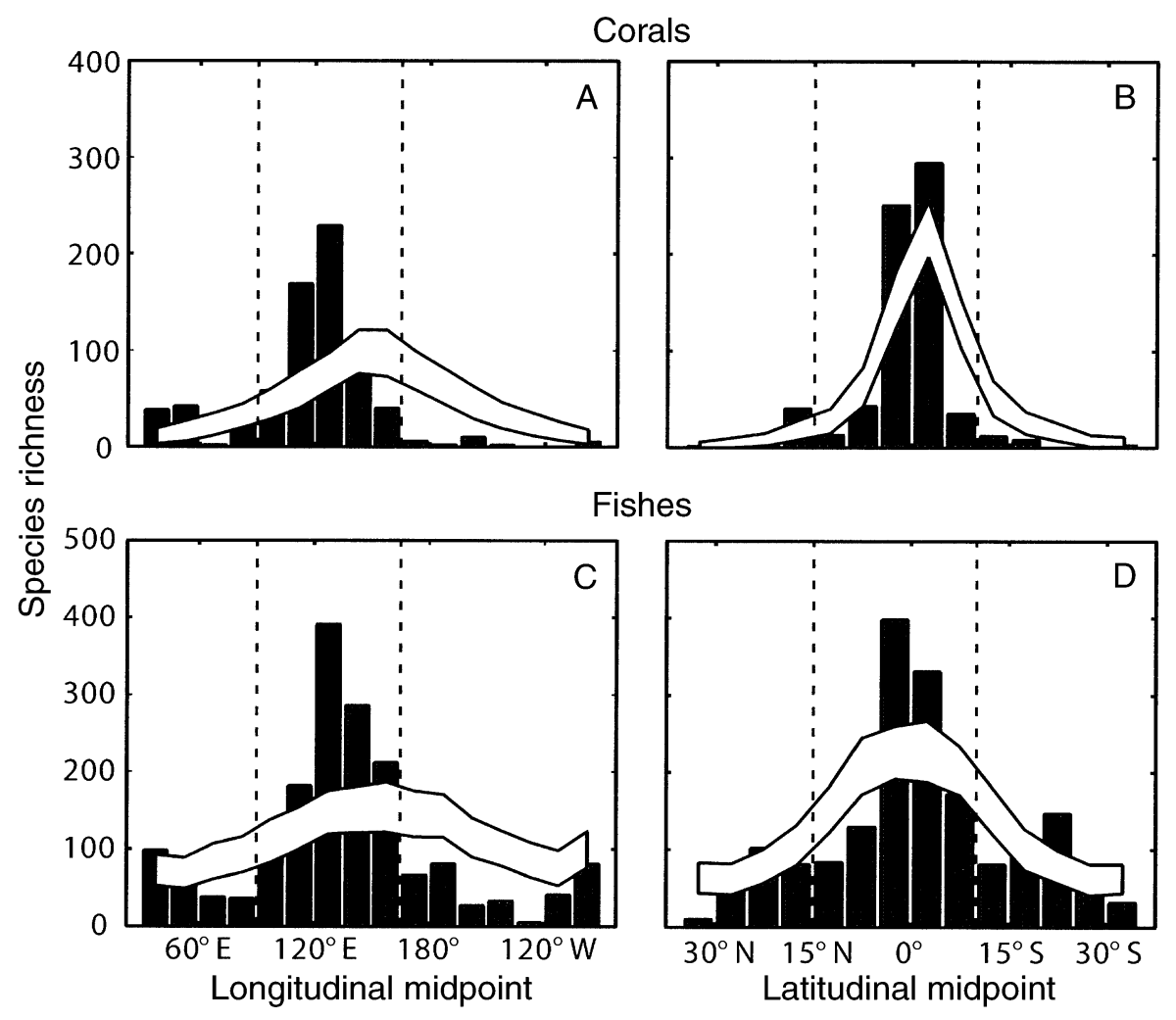

FIG. 5. Observed distribution of range midpoints (bars), and expected distribution based on mid-domain randomizations: (A) corals, longitudinal midpoints; (B) corals, latitudinal midpoints; (C) fish, longitudinal midpoints; and (D) fish, latitudinal midpoints. Enclosed white regions encompass $99 \%$ of randomizations. Vertical dashed lines represent the boundaries of the Indo-Australian Archipelago. In (A) and (C), the leftmost (western) bin includes all midpoints west of $45^{\circ} \mathrm{E}$ (approximately the mouth of the Red Sea and northern Mozambique), while the rightmost bin includes all midpoints east of $105^{\circ} \mathrm{W}$ (central and South America, including the Galápagos Islands). Intervening bins show $15^{\circ}$ intervals. In (B) and (D), the leftmost and rightmost bins represent locations north of $30^{\circ} \mathrm{N}$ and south of $30^{\circ} \mathrm{S}$, respectively. Intervening bins show $5^{\circ}$ intervals.

tered near the limits of the northern (fish and corals) and southern (fish) tropics.

\section{Robustness}

The alternative randomizations yielded predicted species-richness patterns that differed only slightly in detail, with the significance and relative magnitudes of observed deviations nearly identical, with one exception. In the randomization of longitudinal range extents of fishes that constrained range endpoints to fall on shallow-water habitat within the species' observed latitudinal range, the excess of western range endpoints smeared through the IAA (Fig. 4C) was largely predicted by the mid-domain model, and the enrichment of the longitudes of the IAA, while still present, was somewhat diminished (Appendix C).

\section{Discussion}

\section{Indo-Pacific biodiversity gradients}

Our results show that observed patterns of species richness differ significantly from those expected under a mid-domain model. The longitudes corresponding to both the Indo-Australian Archipelago (IAA) center of biodiversity, and the Red Sea/Western Indian Ocean are highly enriched, relative to expectation, while much of the Pacific Ocean is depauperate. Latitudinally, we find that species richness is consistent with the middomain prediction at the equator; but is higher than predicted near the northern (corals and fishes) and southern (fishes) margins of the tropics. Because our model randomizes range location, but uses observed range extents, these differences can be unambiguously attributed to nonrandomness in the spatial distribution of geographic range extents. Moving a step further than earlier mid-domain analyses (see Colwell and Lees 2000 for a review), we compare observed and predicted locations of range endpoints and midpoints, characterizing precisely how the distribution of species in space causes observed species-richness patterns to differ from those expected under a mid-domain model.

Midpoint deviations reveal that the positive speciesrichness anomalies of the longitudes of the IAA and the Red Sea/Western Indian Ocean have different causes. The positive richness anomaly near the western margin of the Indo-Pacific (Fig. 3A) is driven by a tendency for species to extend westward to the African coast 
more frequently than expected by chance alone (Fig. $4 \mathrm{~A}, \mathrm{C})$. This tendency exists for both large-range and small-range species (areas 1 and 2 in Fig. 6A, C). However, because the midpoint excess in the western IndoPacific is small (Fig. 5A, C), relative to the excess of western range limits (Fig. 4A, C), it is clear that the major cause of this diversity anomaly is not endemics (cf. Hughes et al. 2002), but rather species that range from Africa eastward across the Indian Ocean, reaching eastern limits at various points in and beyond the IAA. The positive richness anomaly in the IAA, however, is not driven by large-range species. Large-range species, many of which span over half the domain, would tend to encompass the IAA regardless of their centering, and thus would be incorporated in the mid-domain prediction of species richness for the IAA. Rather, the anomaly is driven by small-range and medium-range species. For corals, this arises from a disproportionate tendency for species to reach their western limits at the western margin of the IAA (area 3 of Fig. 6A). For fish, it arises because species with small and medium ranges are more centered in the IAA than expected (area 4 of Fig. 6C). This clustering of large and small ranges flush against the western side of the domain, and of small and medium ranges centered in the IAA, is responsible for the asymmetric biodiversity gradient, with positive species-richness anomalies in the Indian Ocean and IAA, and negative anomalies throughout the Pacific (Fig. 3A).

Latitudinally, ranges tend to be more centered at the equator than expected, even considering the fact that their range midpoints are geometrically constrained to lie near the domain center (Fig. 5B, D). However, this pattern is driven only by large-range species of both corals and fishes; small-range species are disproportionately centered towards the margins of the tropics (Fig. 6B, D). This directly contradicts the mechanism underlying Rapoport's Rule, according to which smallrange species should be disproportionately centered near the Equator (Stevens 1989, see also Hughes et al. 2002). Given the similarity in midpoint deviations for the two taxa, their very different patterns of speciesrichness deviations (Fig. 3B) is surprising at first. This discrepancy is due to two differences between fishes and corals. Firstly, the equator is not the center of the coral domain (which ranges from $\sim 40^{\circ} \mathrm{S}$ to $35^{\circ} \mathrm{N}$ ), but rather, the center of the coral domain lies south of the Equator. Secondly, corals show a cluster of smallrange species centered in the northern tropics, but not the southern tropics (Fig. 6B); indeed, there is a paucity of corals centered within the Southern Hemisphere (Fig. 5B). As a result, there tend to be more coral species than expected in the northern half of the domain, and fewer than expected in the southern half (Fig. 2B). By contrast, the equator does lie in the center of the fish domain. Because fishes with large ranges are more centered at the mid-domain than expected (Fig. $6 \mathrm{D})$, species richness stays fairly constant through the middle region of the domain, and then begins to drop sharply as the edges of these ranges are crossed (Fig. 2D). Thus, species richness becomes increasingly higher than expected as tropical margins are approached, then drops sharply below expectation beyond them (Fig. 3B).

It is interesting that the longitudes of the IAA are enriched, relative to expectation, but the equatorial latitudes of the IAA are not. For this reason, it is tempting to conclude that the longitudinal species-richness deviation corresponding to the IAA is due to higher-thanpredicted species richness at higher latitudes - north of the Philippines (corals and fishes) and in Australia (fishes), rather than within the IAA itself_-given that those high-latitude locations represent areas that are both latitudinally and longitudinally enriched relative to expectation (Fig. 3A, B). To assess this possibility, we repeated our longitudinal analyses using an eastwest slice of the domain that included only the equatorial latitudes of the IAA (i.e., we analyzed only those species found in the latitudinal region $15^{\circ} \mathrm{N}-10^{\circ} \mathrm{S}$ ). We also repeated our latitudinal analyses using a northsouth slice of the domain that included only the longitudes of the IAA (i.e., we analyzed only those species found in the longitudinal region $90^{\circ} \mathrm{E}-160^{\circ} \mathrm{E}$ ). These yielded results that were virtually identical to the original analyses, with one exception: in the latitudinal analyses, fish no longer exhibited higher-than-predicted species richness in the Northern Hemisphere. A similar north-south slice along the coast of Africa did indicate excess species in the Northern Hemisphere, suggesting that the concentration of fishes in the Northern Hemisphere is principally an Indian Ocean phenomenon. We conclude from these analyses that the IAA is, indeed, longitudinally but not latitudinally enriched. In other words, species ranges are longitudinally clustered over the IAA, but, while latitudinally centered within the IAA, they tend to extend throughout the tropics. As a result, the IAA is not enriched relative to regions directly north and south of it.

\section{Alternative formulations of mid-domain expectation}

Our analyses preserve extent of occurrence, rather than area of occupancy. This is consistent with most one-dimensional mid-domain analyses (e.g., Piñeda and Caswell 1998, Lees et al. 1999, Koleff and Gaston 2001), but differs from that of most two-dimensional analyses, which tend to preserve either actual area of occupancy (number of occupied grid cells), or area encompassed by a geographic range (e.g., Jetz and Rahbek 2001). The highly noncontiguous nature of coral reef habitat led us to favor an analysis that preserved extent of occurrence, as outlined in the Methods and Appendix A. Because shallow-water habitat is not distributed evenly throughout the domain, an area-preserving analysis would clearly produce a different statistical expectation than the extent-preserving analysis conducted here. While a comprehensive comparison of 


\section{Corals}
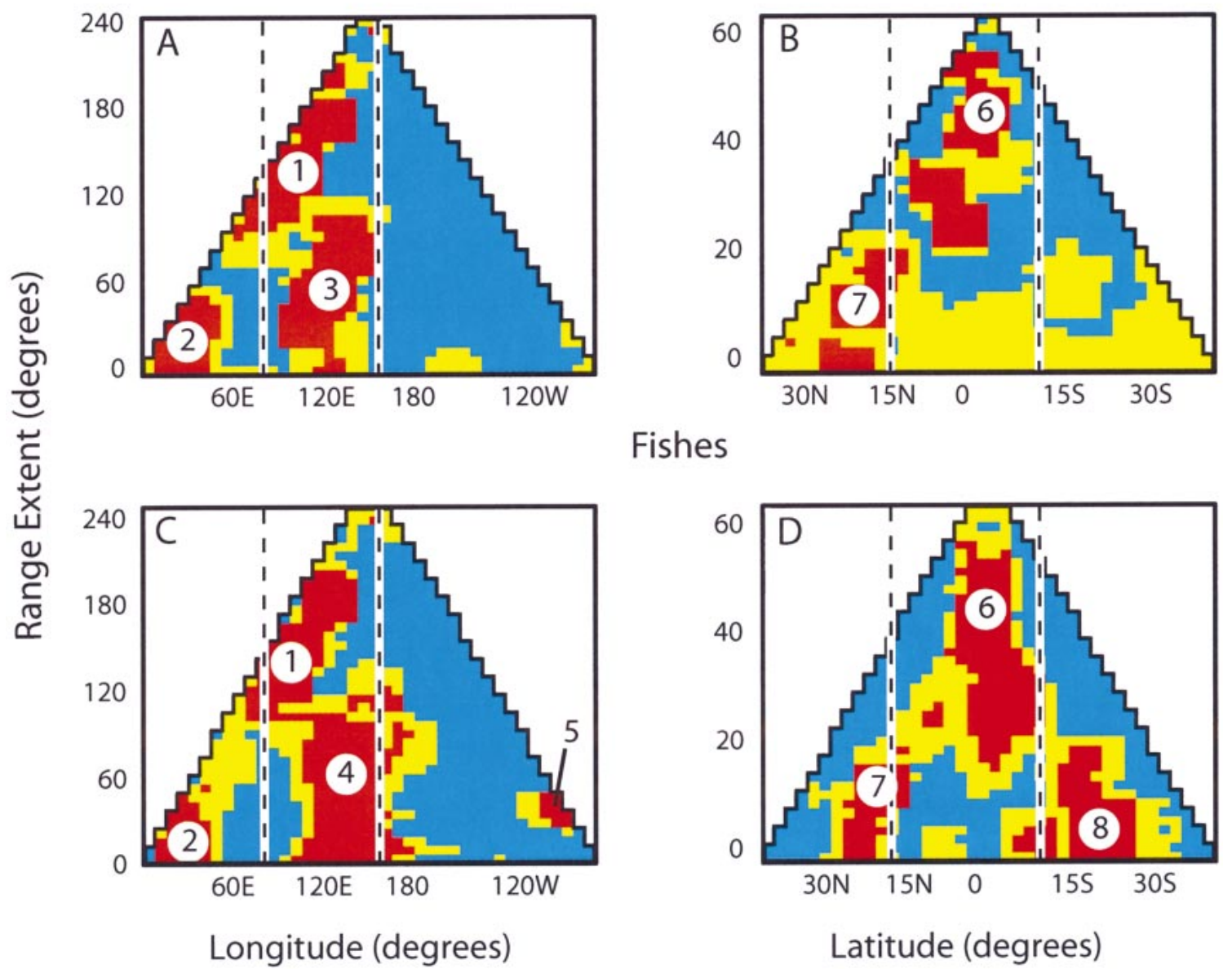

Fishes

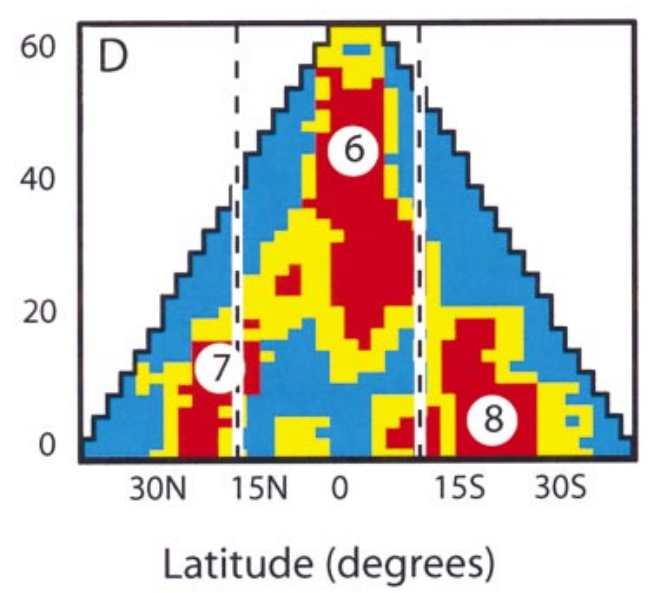

FIG. 6. Midpoint deviation plots: (A) corals, longitude; (B) corals, latitude; (C) fish, longitude; and (D) fish, latitude. Yellow indicates midpoint densities whose values are within $99 \%$ of simulated midpoint densities, while red and pale blue indicate midpoint densities that are above and below these confidence limits, respectively. Vertical dashed lines represent the boundaries of the Indo-Australian Archipelago, and numbers indicate regions of higher-than-expected midpoint density (see Results: Midpoint deviations for explanation). Coarser and finer subdivisions revealed the same eight major features.

the two approaches will have to await such an analysis, the well-known broad-scale patterns of the distribution of coral reefs allow a preliminary assessment of the extent to which the distribution of shallow-water habitat may contribute to the deviations observed in this study.

The spatial distribution of habitat area appears qualitatively consistent with longitudinal deviations in species richness, but not with latitudinal deviations in species richness. Shallow-water carbonate habitat is most abundant in and near the IAA, intermediate in the Indian Ocean, and most scarce across the mid- and eastern Pacific (Spalding et al. 2001). Thus, we would expect an area-preserving randomization to predict a more enriched IAA, and a more rapid increase of species richness from the western margin inwards than from the eastern margin inwards (Fig. 2A, C). Latitudinally, however, shallow-water carbonate habitat availability is overwhelmingly concentrated near the equatorial latitudes of the IAA, and away from latitudes near and beyond the tropical margins (Veron 1995, Bellwood and Hughes 2001). Thus, it is unlikely that the distribution of habitat area could explain why the tropical margins are enriched relative to expectation, while more equatorial latitudes are not (Fig. 3B, D). In particular, the apparent tendency for small-range species to be concentrated near the tropical margins (Fig. 6B, D) seems inconsistent with the distribution of available habitat.

\section{Alternative causes of biodiversity gradients}

Traditionally, explanations of Indo-Pacific biodiversity patterns have focused on explaining decreasing species richness with increasing longitudinal and latitudinal distance from the IAA by invoking environmental factors that would enhance species richness in 
the IAA. We propose th at, instead, these factors should explain the disproportionate tendencies for species ranges to end, or be centered, in particular locations. From this new perspective, it is reasonable to ask whether prevailing hypothesized causes of speciesrichness patterns are consistent with this nonrandomness in species distributions. Specifically, variation in habitat area and complexity (Rosen 1988, Bellwood and Hughes 2001), energy input (Rohde 1992, Fraser and Currie 1996), and patterns of range expansion driven by westward-flowing equatorial currents (Ladd 1960, Jokiel and Martinelli 1992) are three environmental factors proposed to have substantially influenced present-day Indo-Pacific species-richness gradients. Here, we offer a preliminary assessment of the extent to which these factors are consistent with the results of our analyses.

Habitat area may promote speciation or reduce local extinction, leading to higher-than-predicted species richness where habitat area is more abundant, and lower-than-predicted species richness where habitat is less abundant. Thus, its effects are likely to be qualitatively similar to those of an area-preserving randomization analysis. In particular, area effects appear at least partially consistent with longitudinal deviations in species richness, particularly the enriched IAA and the depauperate middle and eastern Pacific (Fig. 3A). However, it is less clear how area could explain latitudinal deviations in species richness, since area is most abundant near the equator, but species-richness deviations increase towards the margins of the tropics (Fig. 3B). In particular, whether area makes the IAA a "cradle" of speciation, or a "museum" due to reduced extinction (Rosen 1988, Bellwood and Wainwright 2002), one would not expect small-range species to be disproportionately centered away from this region, towards the tropical margins (Fig. 6B, D).

Energy supply to coral reef ecosystems has been proposed to promote coexistence (i.e., reduce extinction), and to promote speciation (Rohde 1992, Fraser and Currie 1996). Solar radiation and temperature, two common surrogate variables for energy supply, have been offered as potential explanations for species-richness gradients on coral reefs (Fraser and Currie 1996), given that these factors, like absolute species richness, decrease with latitude. However, like area, decreasing energy supply with increasing latitude does not appear consistent with our finding that, with the exception of corals in the Southern Hemisphere, enrichment relative to expectation grows as one moves from the equator to the margins of the tropics. Longitudinally, we know of no reason to expect solar radiation to be markedly higher in the longitudes of the IAA and Red Sea/Western Indian Ocean than in the eastern Pacific. Temperature seems more promising, as upwelling along the west coast of the Americas would tend to make temperatures colder in the eastern Pacific than at comparable latitudes elsewhere. However, we would not ex- pect the comparatively warm central Pacific to be so depauperate, relative to expectation, if temperature were the principal causal factor (Fig. 3A). Rather, we would expect species to extend readily across the central Pacific, with species richness dropping abruptly between the relatively warm reefs of French Polynesia and the upwelling regions adjacent to the Americas.

A third hypothesis attributes species-richness gradients to a tendency for species to expand their ranges westward more readily than eastward, due to the predominantly westward-flow associated with equatorial currents in ocean basins (Ladd 1960, Jokiel and Martinelli 1992). Superficially, this appears incomplete as an explanation for Indo-Pacific species-richness patterns, given that richness increases to the west across the Pacific Ocean, but not across the Indian Ocean (Fig. $2 \mathrm{~A}, \mathrm{C})$. Thus, the Indian Ocean has been considered an exception to this phenomenon (Jokiel and Martinelli 1992), even though oceanographic models suggest that drifting particles accumulate along the east African coast, as well as within the IAA (Koga and Yabe 1989). However, when the Indo-Pacific is considered as a whole, and the focus shifted from species numbers to the locations of geographic ranges, the Indian Ocean no longer appears inconsistent with an "oceanic gyre hypothesis." If westward-flowing currents drive biodiversity patterns, then ranges should expand more readily westward than eastward across ocean basins from a point of origination. Thus, in the long run, western range limits should accumulate at the African margin of the Indo-Pacific. By contrast, there should be a dearth of eastern range limits reaching the eastern margin of the Indo-Pacific. This is precisely what we observe (Fig. 4A, C). The largest positive anomaly, by far, in either western or eastern endpoints is the accumulation of range endpoints at the western boundary: over 200 species more than expected end their ranges along the African coast, for both corals and fishes. By contrast, there is a large negative anomaly of eastern endpoints in the eastern Indo-Pacific, with corals and fishes instead tending to reach their eastern limits at various points between the eastern IAA and French Polynesia. The IAA, due to its location in between the Pacific and Indian Ocean gyres, might be expected to slow the westward expansion of geographic ranges, and thus show a secondary excess of western range endpoints, consistent with the pattern of drifting particle accumulation exhibited by oceanographic models (Koga and Yabe 1989). Thus, the observed excess of western endpoints in the IAA is also consistent with an oceanic gyre hypothesis (Fig. 4A, C; but see Appendix $\mathrm{C}$ ).

An oceanic gyre hypothesis also offers an explanation for latitudinal patterns (cf. Veron 1995). Specifically, oceanic gyres flow away from the Equator along the coasts of Africa, Asia, and eastern Australia. Moreover, the alongshore Leeuwin current flows away from the equator in western Australia, in spite of the north- 
ward-flowing gyre offshore. Equatorward coastal currents only prevail adjacent to the low-diversity Americas. This prevalence of poleward-flowing coastal currents would tend to cause species originating near the equator to expand their ranges readily north and south, developing large ranges disproportionately centered at the Equator. Conversely, species originating near tropical margins might be expected to have their equatorward extension inhibited, and thus we might expect them to retain small ranges centred away from the equator. Both patterns are apparent in our analyses (areas 6, 7, and 8 in Fig. 6B, D).

\section{Implications}

Historically, explanations for latitudinal and longitudinal species-richness gradients have tended to focus on the relationship between variation in the values of predictor variables (e.g., area, energy) and variation in numbers of species or genera (e.g., Rohde 1992, Rosenzweig 1995, Fraser and Currie 1996, Bellwood and Hughes 2001). Because species range across multiple locations, however, estimates of species richness in different locations are not independent (Hughes et al. 2002, Jetz and Rahbek 2002). This is a particularly salient issue for groups with many large-range species, such as corals and reef fishes. Moreover, geometric constraints on the locations of large ranges imply a marked mid-domain peak in species richness, even in the absence of any environmental gradients (Colwell and Hurtt 1994). Here, focusing on the extent to which observed species-richness patterns deviate from randomized mid-domain expectations fundamentally redefines the Indo-Pacific biodiversity problem. It is these deviations of observed from predicted species richness, or, more precisely, patterns in the distribution of species ranges that give rise to those deviations, that putative environmental causes of species-richness gradients must explain. Unlike raw numbers of species, deviations in species richness do not exhibit a monotonic decrease with increasing distance from the IAA (Fig. 3). In light of that result, it is not surprising that factors that correlate well with numbers of species do not necessarily correlate well with their deviations from a mid-domain prediction.

Alternative hypotheses about the causes of biodiversity gradients are generally based on putative causal relationships between particular environmental variables and the dynamic population processes that give rise to species-richness patterns: speciation, founding of new populations by dispersal (range expansion), and population extinction (range contraction). These relationships may not give rise to straightforward correlations between species richness and those same environmental variables, as most earlier approaches implicitly assume. This is especially true when species ranges tend to span many locations, and thus the likelihood that a species' range encompasses one location depends upon whether or not it is present at nearby locations. For these reasons, the development of analyses that rigorously and quantitatively assess the extent to which alternative hypotheses can explain nonrandomness in the distribution of species ranges poses a difficult conceptual challenge. Nevertheless, we believe that this is a challenge worth confronting. Our focus on quantifying nonrandomness in the distribution of species ranges offers a fresh perspective on the nature and causes of Indo-Pacific biodiversity gradients. Fully exploiting this perspective has the potential to dramatically change our picture of how factors such as area, energy supply, and ocean circulation influence global species-richness patterns.

\section{ACKNOWLEDGMENTS}

We are grateful to N. Gotelli, W. Jetz, K. Lyons, and P. Munday for thoughtful, constructive comments on an earlier version of this manuscript. We thank Klaas Hartmann for programming randomization algorithms and producing figures, and Marie Kospartov and Sula Blake for additional assistance with figures. P. Jokiel kindly shared a translation of an important Japanese manuscript. The Australian Research Council and James Cook University provided financial support. This is contribution number 67 of the Centre for Coral Reef Biodiversity (CCRB), and contribution number 195 of the Coral Ecology Group at James Cook University.

\section{Literature Cited}

Bellwood, D. R., and T. P. Hughes. 2001. Regional-scale assembly rules and biodiversity of coral reefs. Science 292: $1532-1534$.

Bellwood, D. R., and P. C. Wainwright. 2002. The history and biogeography of fishes on coral reefs. Pages 5-32 in P. F. Sale, editor. Coral reef fishes: dynamics and diversity in a complex ecosystem. Academic Press, Amsterdam, Netherlands.

Colwell, R. K., and G. C. Hurtt. 1994. Nonbiological gradients in species richness and a spurious Rapoport effect. American Naturalist 144:570-595.

Colwell, R. K., and D. C. Lees. 2000. The mid-domain effect: geometric constraints on the geography of species richness. Trends in Ecology and Evolution 15:70-76.

Fraser, R. H., and D. J. Currie. 1996. The species richnessenergy hypothesis in a system where historical factors are thought to prevail: coral reefs. American Naturalist 148: $138-159$.

Gaston, K. J., and T. M. Blackburn. 2000. Pattern and process in macroecology. Blackwell Science, Oxford, UK.

Hughes, T. P., D. R. Bellwood, and S. R. Connolly. 2002. Biodiversity hotspots, centers of endemicity, and the conservation of coral reefs. Ecology Letters 5:775-784.

Huston, M. 1994. Biological diversity: the coexistence of species on changing landscapes. Cambridge University Press, Cambridge, UK.

Jetz, W., and C. Rahbek. 2001. Geometric constraints explain much of the species richness pattern in African birds. Proceedings of the National Academy of Sciences (USA) 98 : $5661-5666$

Jetz, W., and C. Rahbek. 2002. Geographic range size and determinants of avian species richness. Science 297:15481551.

Jokiel, P., and F. J. Martinelli. 1992. The vortex model of coral reef biogeography. Journal of Biogeography 19:449_ 458.

Jones, G. P., M. J. Caley, and P. L. Munday. 2002. Rarity in coral reef fish communities. Pages 81-101 in P. F. Sale, editor. Coral reef fishes: dynamics and diversity in a complex ecosystem. Academic Press, Amsterdam, Netherlands. 
Koga, T., and M. Yabe. 1989. NHN Chiku Osen (Polluted Earth). Volume 2. [In Japanese.] Nihoiihose Shuppan Kyoka, Tokyo, Japan.

Koleff, P., and K. J. Gaston. 2001. Latitudinal gradients in diversity: real patterns and random models. Ecography 24: 341-351.

Ladd, H. S. 1960. Origin of the Pacific island molluscan fauna. American Journal of Science 258A:137-150.

Lees, D. C., C. Kremen, and L. Andriamampianina. 1999. A null model for species richness gradients: bounded range overlap of butterflies and other rainforest endemics in Madagascar. Biological Journal of the Linnean Society 67:529584.

Lyons, S. K., and M. R. Willig. 1997. Latitudinal patterns of range size: methodological concerns and empirical evaluations for New World bats and marsupials. Oikos 79:568580 .

McManus, J. W. 1985. Marine speciation, tectonics, and sealevel changes in southeast Asia. Pages 133-138 in C. Gabrie and M. Harmelin Vivien, editors Proceedings of the Fifth International Coral Reef Congress (Tahiti, 1985). Volume 4. Antenne Museum-Ephe, Moorea, French Polynesia.

Palumbi, S. R. 1997. Molecular biogeography of the Pacific. Coral Reefs 16:S47-S52.

Paulay, G. 1997. Diversity and distribution of reef organisms. Pages 298-373 in C. E. Birkeland, editor. Life and death of coral reefs. Chapman and Hall, London, UK.

Pianka, E. R. 1978. Evolutionary ecology. Second edition. Harper and Row, New York, New York, USA.

Piñeda, J., and H. Caswell. 1998. Bathymetric species-diversity patterns and boundary constraints on vertical range distributions. Deep Sea Research II 45:83-101.

Rahbek, C. 1997. The relationship among area, elevation, and regional species richness in neotropical birds. American Naturalist 149:875-902.
Roberts, C. M., C. J. McClean, J. E. N. Veron, J. P. Hawkins, G. R. Allen, D. E. McAllister, C. G. Mittermeier, F. W. Schueler, M. Spalding, F. Wells, C. Vynne, and T. B. Werner. 2002. Marine biodiversity hotspots and conservation priorities for tropical reefs. Science 295:1280-1284.

Rohde, K. 1992. Latitudinal gradients in species diversity: the search for the primary cause. Oikos 65:514-527.

Rosen, B. R. 1988. Progress, problems and patterns in the biogeography of reef corals and other tropical marine organisms. Helgoländer Meeresuntersuchungen 42:269-301.

Rosenzweig, M. L. 1995. Species diversity in space and time. Cambridge University Press, Cambridge, UK.

Spalding, M. D., C. Ravilious, and E. P. Green. 2001. World atlas of coral reefs. University of California Press, Berkeley, California, USA.

Stehli, F. G., and J. W. Wells. 1971. Diversity and age patterns in hermatypic corals. Systematic Zoology 20:115-126.

Stevens, G. C. 1989. The latitudinal gradient in geographical range: how so many species coexist in the tropics. American Naturalist 133:240-256.

Tomczak, M., and J. S. Godfrey. 1994. Regional oceanography: an introduction. Pergamon, Oxford, UK.

Veech, J. A. 2000. A null model for detecting nonrandom patterns of species richness along spatial gradients. Ecology 81:1143-1149.

Veron, J. E. N. 1995. Corals in space and time: the biogeography and evolution of the Scleractinian. University of New South Wales Press, Sydney, New South Wales, Australia.

Wallace, A. R. 1878. Tropical nature and other essays. Macmillan, London, UK.

Willig, M. R., and S. K. Lyons. 1998. An analytical model of latitudinal gradients of species richness with an empirical test for marsupials and bats in the New World. Oikos 81: 93-98.

\section{APPENDIX A}

An explication of the randomization method is available in ESA's Electronic Data Archive: Ecological Archives E084052-A1.

\section{APPENDIX B}

A description of alternative randomizations is available in ESA's Electronic Data Archive: Ecological Archives E084052-A2.

\section{APPENDIX C}

The results of conditional, habitat-constrained randomizations are available in ESA's Electronic Data Archive: Ecological Archives E084-052-A3. 